\title{
DoItRight: An Arabic Gamified Mobile Application to Raise Awareness about the Effect of Littering among Children
}

\author{
Ayman Alfahid ${ }^{1}$, Hind Bitar ${ }^{2}$, Mayda Alrige ${ }^{3}$, Hend Abeeri $^{4}$, Eman Sulami $^{5}$ \\ Computer Science, Majmaah University, Majmaah, Saudi Arabia ${ }^{1}$ \\ Information Systems, King Abdulaziz University, Jeddah, Saudi Arabia ${ }^{2,3,4,5}$
}

\begin{abstract}
Littering contributes significantly to environmental pollution. Previous studies have noted that children are more likely to litter than adults. This target age group can be easily reached through mobile applications and games. Therefore, this study aims to investigate the effect of a gamified application in raising awareness on the effect of littering in the environment. We developed a gamified app, called DoItRight to promote an environment friendly behavior and improve the littering behavior of children. The DoItRight app is in Arabic language and targets children between 5 and 13 years old. It is a gamified application that enables kids to learn the importance of picking up litters and dropping it in trash cans. The app was evaluated using the System Usability Scale (SUS) standardized instrument which was administered on the target audience. The results of the evaluation showed that the DoItRight app has an SUS score of 93.25 which represents an $A+$ grade and a percentile range of 96 to 100. This indicates that the DoItRight app is technically usable and can potentially serve the purpose of increasing kids' awareness about the downsides of littering on the environment.
\end{abstract}

Keywords-Littering; mobile application; gamification; children intention; raise awareness; behavior change Saudi Arabia

\section{INTRODUCTION}

Over the years, human practices have caused several environmental hazards which can inhibit future growth and wellbeing. The collective impact of human behaviors on the environment further threatens our ecosystem [1] - [3]. One of such behaviors is littering which can either be accidental or intentional. Therefore, identifying factors responsible for high rate of littering is critical to design an efficient litteringreduction intervention. A study carried out in 2020 used the Motivation, Opportunity, Ability and Behavior (MOAB) framework to examine the individual littering behaviors of Saudi citizens. It conducted twenty-five semi-structured interviews on individuals between 20 and 40 years in Saudi Arabia. The outcomes of the study showed that the lack of knowledge, at the individual level, influences littering behavior. Other factors found include social norms and the built environment. Therefore, any plan to reduce littering must include the implementation of an individual-level strategy [4].

Additional individual and environmental factors influencing littering behavior in Saudi Arabia have also been unraveled. Recent research observed 362 individuals and their surrounding environments for twelve days in Saudi Arabia [5]. Findings revealed that littering rates were higher in environments that were less attractive and where trash cans were far from individuals. Also, the study found out that younger people are more likely to litter than older people.

Meanwhile, the global increase in the use of smartphones has led to the emergence of mobile applications being developed and deployed for education and enlightenment on several issues including environment and health (for instance, mhealth systems) [6]. Also, researchers have acknowledged the effectiveness of mobile applications in educating children. Therefore, the use of a mobile app to raise children's awareness of the threats posed by littering can prove to be an effective litter-reduction strategy [7]. Therefore, this research designs, and evaluate an Arabic gamified mobile application following user-centered design approach, called "DoItRight" to increase children's awareness and intent towards littering. It targets children from 5 to 13 years.

\section{A. User-Centered Approach}

As the name implies, a user-centered design (UCD) approach puts the user at the center of the design process. That is, the designer of a product or application considers the needs, limitations, and interests of the end users and designs a product that meets those needs. Having an understanding of who we design for, what they need, and their environmental circumstances is an effective way to ensure that a product or design is successful. It is also a good way of preventing a bad design that can potentially frustrate users [8]. The UCD approach involves understanding user context, defining requirements, developing solutions, and evaluating the outcomes with respect to the users' requirements and contexts [9]. In this study, we adopted a user-centered design approach to develop the DoItRight application.

The rest of this paper is organized as follows: Section 2 explores the related work in the existing literature. Section 3 presents the methodology adopted in conducting the research. Results are presented and discussed in Section 4 while Section 5 concludes the paper and sets the direction for future work.

\section{RELATED WORK}

\section{A. Improving Children's Littering Behavior}

Education in different forms can be used to improve children's behavior towards the environment. Reference [10] reported outcomes of the "We Love Reading Program" that 
leveraged the reading of social stories to address littering and environmental issues across different communities in Jordan. The results indicated that the program improved children's knowledge of littering issues and created a positive change in the behavior of the kids.

The author in [11] explored 5th grade children's solutions to littering and environmental pollution using the kids' drawings. Forty children at the age of 10 and 11 years, including 25 girls and 15 boys, completed drawings to provide solutions and future plans to littering and environmental pollution. The study utilized three themes to analyze the drawings; these include persuasion, physical action, and political action. However, most of the children found it difficult to visually express their thoughts on persuasion and political action. The major solutions common in the drawings include collecting litters, dropping litters in the trash can, and planting saplings [11]. The children in the research knew littering is a great environmental challenge that needs to be addressed with more than one strategy.

In a recent publication [12], researchers echoed the fact that a proper upbringing of a child potentially represents a great beginning towards developing a good littering behavior. A survey administered by the study recorded responses from 2,349 individuals. The outcome of the survey showed that lowincome households need support in terms of quality education and disposal facilities while high-income households need support in terms raising awareness on the problems of littering. Though self-initiative and parental guidance constitute a good approach towards improving the littering behavior of children, they are not sufficient [12]. The researchers suggested that future research should explore the effectiveness of religious education on the littering behavior of children.

To find a lasting solution to the littering problem, it is important to understand the factors that motivate people to dump litters in the environment or in the bin [13]. Researchers [13] explored these motivating factors by conducting a survey that asked people to indicate what motivates them to drop litters in the bin. An analysis of the survey responses divided the motivators into intrinsic and extrinsic motivators. The results showed that sense of morals, ethics and upbringing were the highest-level intrinsic motivators while the highestlevel extrinsic factors were the presence of kids, being in a clean place, and recycling programs. Therefore, to create a successful anti-littering campaign in developing countries, stakeholders should combine intrinsic and extrinsic motivators. The study [13] noted that, for the intrinsic motivators, authorities should remind people of their core values, morals and ethics. For the extrinsic factors, authorities should create a convenient infrastructure, recycling programs, rewards, and penalties.

\section{B. Use of Digital Technologies to Address Littering Issues}

Some existing research works have explored the possibility of leveraging digital technologies and mobile applications to improve the littering behavior of children. Researchers [14] concluded that it is extremely important to raise children's awareness towards making sustainable choices when buying, using or dismissing products. One of the solutions proposed was the development of Contact from the future, a digital game focusing on plastic pollution education of children. After defining the requirements and objectives of the game, the study designed and developed the game application. The ultimate goal was to raise awareness and stimulate pro-environmental behaviors in children.

Another study [15] identified inadequate environmental education or awareness at the early age as a critical cause of littering and environmental problems. To address the issue, the study developed an Android-based sorting waste game to teach children the different kinds of waste, (i.e., organic and inorganic) and the appropriate litter box to drop each waste. The Waterfall Development Model was adopted meaning that the development of the sorting waste game followed a 4-step process including analysis, design, implementation, and testing. The functionality of the game was tested and the outcome indicated that the game is user friendly, runs smoothly on Android, and can serve as an effective medium of gaining environmental education.

Also, an exploratory study [16] was conducted to understand how children's use of digital technology, especially iPads, can impact outdoor environmental education programs. In particular, iPads were integrated into water quality education for 5 th grade children. The qualitative observations obtained from the study were analyzed and seven major themes emerged. They include children's reaction to mobile devices, digital natives versus immigrants, group interactions, mobile devices in the hands of kids, instruments for learning, nature prevails, and introduction of mobile devices. These themes offered new insights to understand best practices for technology integration into littering and environmental education.

The author in [17] examined the effectiveness of web-based animation videos in the environmental education of elementary school pupils. The research [17] developed web-based animation video pages for climate change, waste recycling, mangrove forest, ozone depletion, and biodiversity. The impact of the intervention was measured via pupils' level of natural curiosity, improvement in environmental awareness, and the pupils' level of pro-conservation values. The outcome of the evaluation revealed that the use of web-based animation videos has positive effect on pupils' littering and environmental education.

Moreover, other researchers [18] have equally emphasized the urgent need to consider the use of virtual and augmented realities, videoconferencing, and mobile apps to engage primary school children with the ultimate goal of preventing littering and restoring the environment. The research [18] indicated that these technologies can capture children's interest while enabling them to learn important practices in preventing littering and protecting the environment. Quick Response (QR) codes are another example of a technology that can be integrated into mobile learning technology in littering education [19]. When attached to an object, the QR codes can add a layer of digital functionality thereby empowering users of mobile devices to access information without any restriction. 


\section{METHODOLOGY}

\section{A. DoItRight App Intervention Design and Development}

We followed four main stages in the design and development of the DoItRight App:

1) Understanding user context

2) Obtaining requirements from parents and families of children who are target audience of the proposed application.

3) Creating a prototype of the app features based on the requirements collected in stage 2 as well as a review of similar mobile applications in the market.

4) Deploy and test the DoItRight app for evaluation; create a formative evaluation protocol to get feedback that can be used to further refine the app.

In stage 1 , we determined the target audience in terms of who they are, their access to smartphones, age group, level of education etc. In stage 2 , we administered a survey filled by parents and families to enable us to identify and define user requirements while also exploring the operational feasibility of the proposed DoItRight app.

In stage 3 , the team reviewed existing mobile applications in the market with similar features and target audience to DoItRight app. The team also developed all necessary features to meet the requirements obtained in stage 2 and fill the gaps in the existing similar mobile applications. In stage 4 , the team prepared and administered a survey on children between 5 and 13 years (target audience) to evaluate the usability, reliability, and response time of the DoItRight app. The design and development stages of the DoItRight app can be seen in Fig. 1.

\section{B. Requirements: Increasing Children Awareness and Intention about Littering}

In stage 1, preliminary studies and review of the existing literature indicate that kids are more likely to litter than adults. These children also have little awareness on the consequences of littering. Therefore, the target audience of this study is determined to be 5 to 13 years. In stage 2, we defined a survey to obtain user requirements of the target audience. The questionnaire was administered using Google Forms, distributed via WhatsApp and filled by parents and families of the target audience. In total, 161 responses were recorded.

- Demography of Participants: $87.6 \%$ of participants are female while $12.4 \%$ are male. $20.5 \%$ of them are less than 20 years, $51.6 \%$ are between $20-40$ years, and $28 \%$ are over 40 years. Also, $46 \%$ of the participants have children with the age of 5 years.

- Results: $90.7 \%$ of participants reported facing the problem of littering in different public places, thereby confirming the significance of the littering problem that the DoItRight app aims to address. Also, $16.8 \%$ of the respondents reported that their children have bad littering behavior and throw wastes on the floor rather than the trash can, even at home. When asked about the causes of littering, 39.1\% chose "lack of education", $54.5 \%$ chose "no responsibility" and $6.4 \%$ chose "others". This indicates that the lack of adequate awareness (education) contributes significantly to the menace of littering. In addition, $98.8 \%$ of participants are willing to allow their children use a mobile application that can increase their level of awareness and intent on littering. Also, 98.8\% will appreciate a solution that is highly interactive, can improve the children's motivation towards dumping litters correctly and can help them apply whatever they learn.

\section{DoItRight App Description}

In stage 3, a prototype of the DoItRight app was developed while ensuring that there the features address the user requirements identified in stage 2 . The mapping of the requirements to the features and the anticipated techniques to increase children's awareness and intention is provided in Table I. The DoItRight app is an education gaming mobile application targeting Saudi children between the age of 5 and 13 years. To access the application, a child needs to create an account which includes providing a username and creating a password of not less than 8 characters. The game consists of 3 levels; each level has specific tasks.

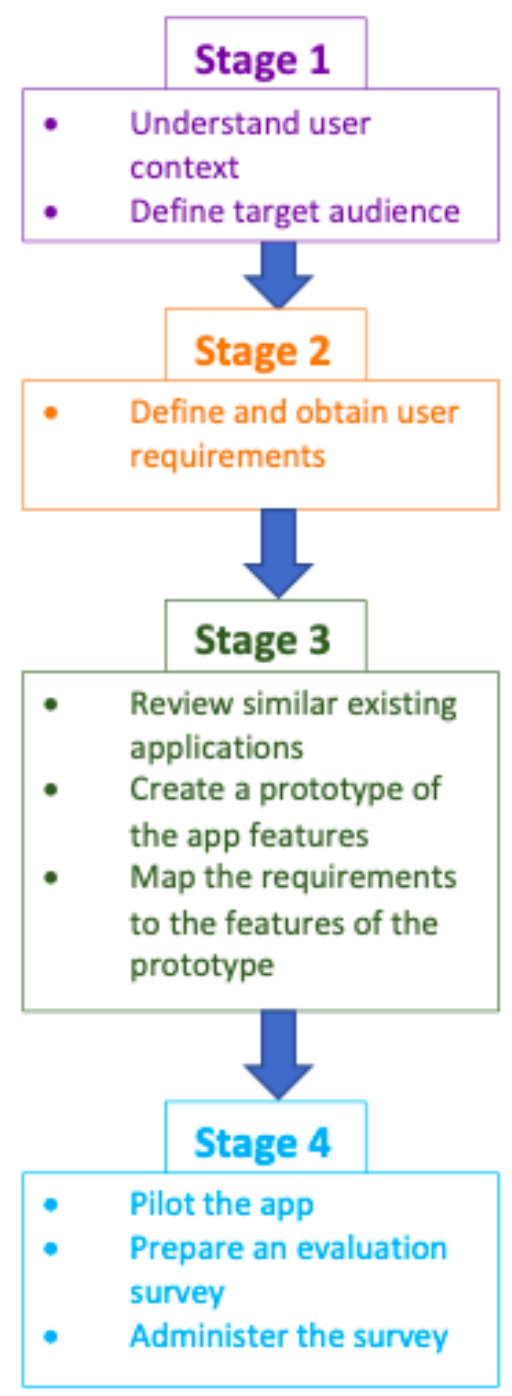

Fig. 1. Shows the Major Highlights of all the Four Stages in the Design and Development of the DoItRight App. 
TABLE I. MAPPING USER REQUIREMENTS TO DOITRIGHT APP FEATURES AND AWARENESS/INTENTION TECHNIQUES

\begin{tabular}{|l|l|l|}
\hline User requirements & App features & $\begin{array}{l}\text { Increasing } \\
\text { awareness and } \\
\text { intention } \\
\text { techniques }\end{array}$ \\
\hline $\begin{array}{l}\text { A means of raising } \\
\text { awareness on } \\
\text { littering }\end{array}$ & $\begin{array}{l}\text { Levels 1 to 3 of the game, food } \\
\text { items in the reward and } \\
\text { evaluation stage of the game }\end{array}$ & $\begin{array}{l}\text { Instruction on } \\
\text { how to perform } \\
\text { the behavior }\end{array}$ \\
\hline Highly interactive & $\begin{array}{l}\text { Use of visuals (videos and } \\
\text { pictures), sounds, texts }\end{array}$ & $\begin{array}{l}\text { Information } \\
\text { provision }\end{array}$ \\
\hline $\begin{array}{l}\text { Motivation for } \\
\text { children to drop } \\
\text { litters appropriately }\end{array}$ & $\begin{array}{l}\text { Food reward after level 3 (candy, } \\
\text { banana, juice, and chips), stars, } \\
\text { accumulation of points }\end{array}$ & Rewarding \\
\hline $\begin{array}{l}\text { Ability to apply } \\
\text { what is learnt }\end{array}$ & $\begin{array}{l}\text { Evaluation task on the empty } \\
\text { candy bag, banana peel, empty } \\
\text { can and empty chips bag on the } \\
\text { floor }\end{array}$ & $\begin{array}{l}\text { Feedback on the } \\
\text { behavior; } \\
\text { prompts }\end{array}$ \\
\hline $\begin{array}{l}\text { Understanding the } \\
\text { importance of good } \\
\text { littering behavior }\end{array}$ & $\begin{array}{l}\text { Videos displaying positive and } \\
\text { negative impacts of varying } \\
\text { actions on the environment }\end{array}$ & $\begin{array}{l}\text { Feedback on the } \\
\text { behavior }\end{array}$ \\
\hline
\end{tabular}

1) First level (the beach)

- The game starts with a child carrying a bag in the beach.

- The child must collect $50 \%$ of the litter at the beach.

- In this first level, the child's speed will be medium.

2) Second level (the park)

- The second level starts with a child carrying a bag in a park.

- The child must collect $50 \%$ of the litter in the park.

- Here, the child's speed will be faster than the first level's speed.

3) Third level (the city streetss)

- The third level starts with a child carrying a bag in the city streets.

- The child must collect $50 \%$ of the litter in the streets.

- The child's speed will be faster than the speed in the second level.

- Throwing the dirtbag into the waste bin completes level 3 while the game proceeds to the reward and evaluation stage.

For completing all three levels successfully, the child is rewarded with food which includes a candy, banana, a can of juice, and a bag of crispy chips. While the food items serve as a reward for a job well done in levels 1 to 3 , they are equally serving as a means to assess the child's level of awareness about littering having played the game. Therefore, after eating the food, the empty candy bag, banana peel, empty can, and empty chip bag are all thrown on the floor. Afterwards, a creative character will appear on the screen to ask the child a question; "what are you going to do now?" If the child picks up the litters and drop in the waste bin, he has passed the test. A congratulatory message will appear. However, if he did not drop the litters in the waste bin after 10 seconds, he will lose and repeat the game.

\section{Key Highlights of the DoItRight App}

1) Methods of collecting points: In each level, the child gets the maximum points obtainable if he collects the highest possible amount of litter that comes his way in the course of the game and successfully drops them all in the waste bin before the end of the level.

2) Difficulty: The child's walking speed will increase after each level, potentially allowing him to collect all the litters before reaching the waste bin.

3) Motivation: Aside from the food reward given to the child at the end of level 3, encouraging sound and objects will appear during the child's walk e.g., stars or clapping sound.

4) Videos at the end of each level: The first video congratulates the child who gets the required number of points and scales through the evaluation test. The video will show the positive impact of the child's act on the environment. Then, it also shows the negative effects of leaving litters on the floor.

The second video shows the child who did not get the required number of points or who did not pass the evaluation test. It shows the negative effects of the litter on the environment. Then, it ends with the positive impact of collecting litters and throwing them in the appropriate waste bin. This helps the child to visualize the positive change his efforts could have brought to the environment if he had dropped the litters in the waste bin.

5) Activity diagram: An activity diagram shows the flow of the interaction between a system and the user in a sequential order. The activity diagram for the DoItRight app is shown in Fig. 2.

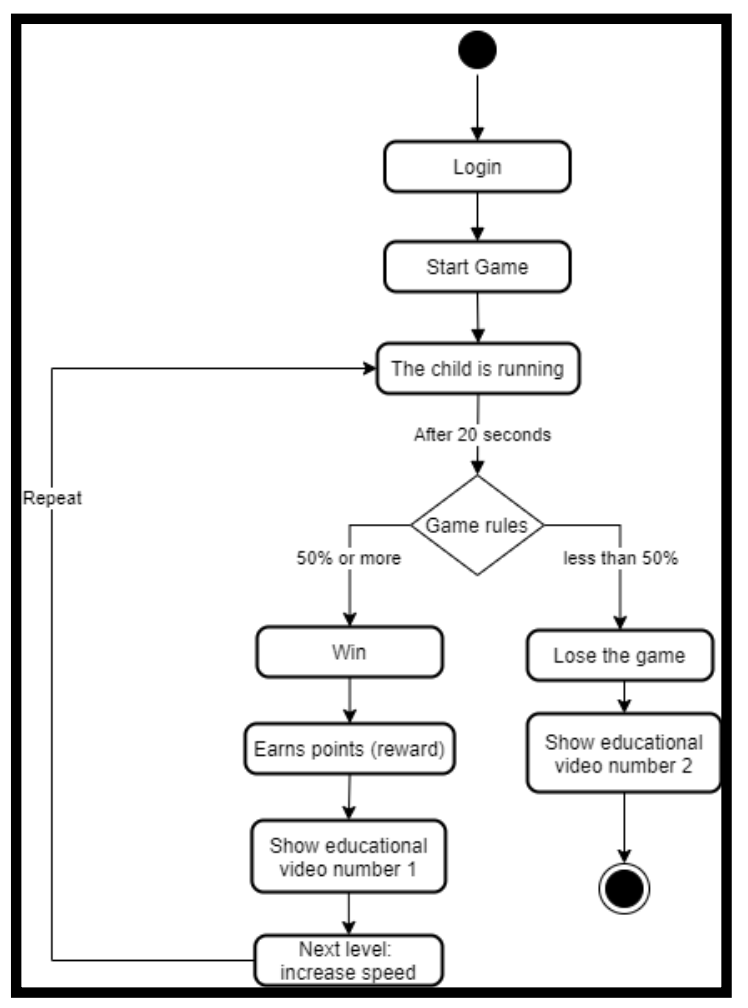

Fig. 2. The Activity Diagram of the DoItRight App Showing the Flow of Interaction between the System and the user. 
6) User interface: The Graphics User Interface of the DoItRight app was designed using JustInMind. Some of the snapshots of the interface like welcome page, education video page, and game page are shown in Fig. 3-5.

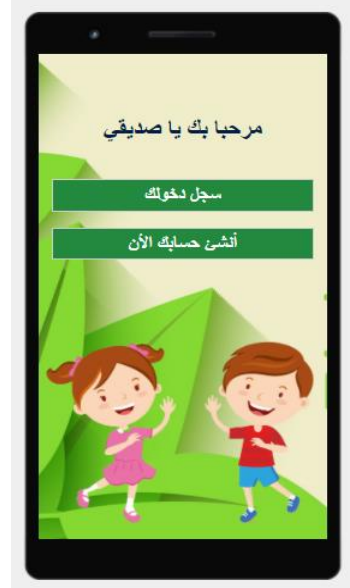

Fig. 3. A user Interface that shows the Welcome Page of DoItRight App.

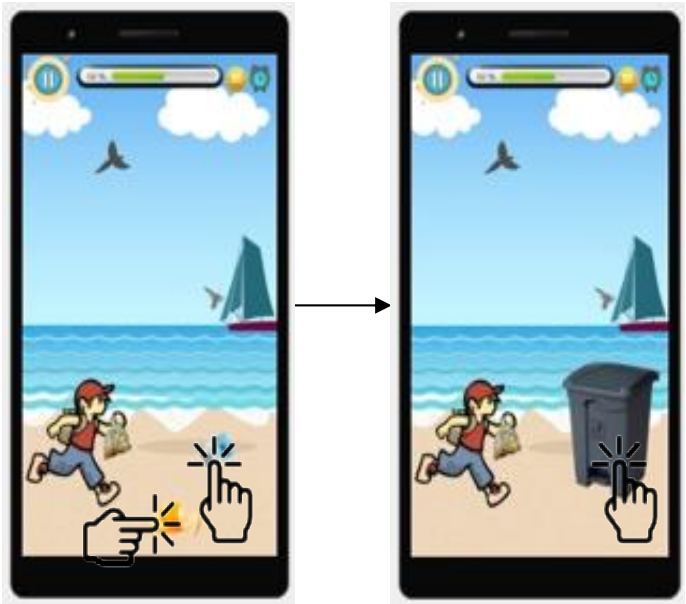

Fig. 4. Two user Interfaces with the First One showing 50\% Progress made in the Level 1 of the Game and the Second Interface Showing a Child Throwing the Dirtbag into the Waste bin.

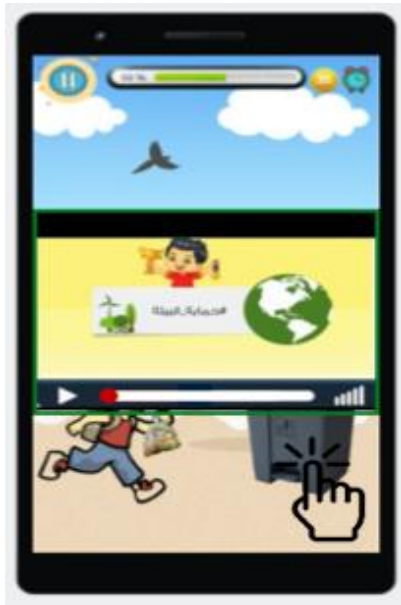

Fig. 5. The Interface Shows an Educational Video on the Positive Environmental Impact of Dropping Litters in the Waste Bin.

\section{E. Testing and Evaluation}

To test and evaluate (stage 4 of UCD) the effectiveness of the DoItRight app, three techniques were implemented namely unit testing, integration testing, and usability testing. These steps help to test the application for quality assurance, both technically and in terms of user satisfaction.

1) Unit testing: Unit testing is a software testing method by which each part of an application (known as a unit) is tested to ensure it is fit for use and meets the desired goal. Part of the objective is also to identify and solve any problems that may arise [20]. In the DoItRight app, we performed unit testing for several units of the app, such as the signup function and the login functions. In the login unit testing, for instance, we tested several cases like leaving the username and password fields blank, filling the password field alone, filling username field alone, and filling correct username and password in their respective fields.

2) Integration testing: While unit testing involves testing a part of the application, integration testing involves testing a combination of units. It is carried out to check if specific units of an application work and interact together as expected. It is usually carried out after the unit testing [21]. For the DoItRight app, we implemented seven different integrated testing objectives. The results are shown in section 4.0.

3) Usability testing: Here, we adopted the Arabic version of System Usability Scale (SUS) which is known as the most popular and widely used standardized questionnaire for assessing perceived usability [22]. SUS consists of a 10-item questionnaire; each question has five response options which include strongly agree, agree, neutral, disagree, and strongly disagree [23]. This evaluation was carried out to determine whether the DoItRight app meets the needs and expectations of the major stakeholders who are the target audience. The questionnaire was deployed using Google Forms. The ten questions include the following:

i. I think that I would like to use this system frequently.

ii. I found the system unnecessarily complex.

iii. I thought the system was easy to use.

iv. I think that I would need the support of a technical person to be able to use this system.

v. I found the various functions in this system were well integrated.

vi. I thought there was too much inconsistency in this system.

vii. I would imagine that most people would learn to use this system very quickly.

viii. I found the system very cumbersome to use.

ix. I felt very confident using the system.

x. I needed to learn a lot of things before I could get going with this system.

Ten children between the age of 5 and 13 were selected as participants in this survey. The full consent of the parents was secured before selecting a child or administering the questionnaire. As per the demography of study participants, we recorded 7 boys with age ranging from 5 to 13 years and 3 girls 
with age ranging from 8 to 13 . Age 8, 11, and 13 recorded 2 children each while age $5,6,7$, and 10 recorded one child each.

\section{RESULTS AND DISCUSSION}

The outcome of the integrated testing implemented on the DoItRight app is shown in Table II. As it can be seen in the table, all the test cases gave positive results.

After analyzing the responses recorded for the System Usability Scale questions, the following results were obtained:

i. $30 \%$ strongly agreed that they would like to use the DoItRight app frequently, $50 \%$ agreed while $20 \%$ were neutral.

ii. $80 \%$ of respondents strongly agreed that the system was easy to use while $20 \%$ agreed.

iii. $30 \%$ strongly agreed that they found the various functions in the system well integrated, $60 \%$ agreed and $10 \%$ were neutral.

iv. $70 \%$ strongly agreed that they would imagine that most people would learn to use the system very quickly; 30\% agreed.

v. $60 \%$ strongly agreed that they felt confident using the system while $40 \%$ agreed.

vi. All respondents strongly disagreed that the system was unnecessarily complex or that there was too much inconsistency in it or that the system was too cumbersome to use or that they needed to learn a lot of things before they could get going with the system. They all strongly disagreed!

vii. $90 \%$ strongly disagreed that they would need the support of a technical person to be able to use this system while $10 \%$ agreed.

Also, based on the responses, we computed the SUS score for the DoItRight app. The SUS score of the app was found to be 93.25. This falls between 84.1 and 100 on the Sauro-Lewis grading scale for SUS scores [24]. Therefore, the DoItRight app falls into the grade A+ category and a percentile range of 96 to 100 on the Sauro-Lewis. As a result, it can be concluded that the DoItRight app is highly acceptable among users.

There are some existing apps that have some similarities with the DoItRight app. Such applications include Watten Games, Garbage Truck, Trash Stash and Littering Game. A comparative analysis between the DoItRight app and the four identified similar apps revealed that the DoItRight app offers better and superior features to address the challenge of littering than any of the other four applications. It supports Arabic language, has several levels and scenarios, rewards players with points, and provides realistic images of characters. This puts the DoItRight application in a position to change the behavior and intent of children towards littering in Saudi Arabia, much better than any other application.

TABLE II. RESULTS FROM THE INTEGRATED TESTING CARRIED OUT ON DOITRIGHT APP

\begin{tabular}{|c|c|c|c|c|}
\hline Test Case ID & Test Case Objectives & Test Case Description & Expected Results & Remark \\
\hline 1 & $\begin{array}{l}\text { To check the interface link between the } \\
\text { first page and the signup page. }\end{array}$ & $\begin{array}{l}\text { The user clicks the "أنشأ حسابك button. } \\
\text { الان" }\end{array}$ & $\begin{array}{l}\text { DoItRight directs the user to the signup } \\
\text { page. }\end{array}$ & Positive test \\
\hline 2 & $\begin{array}{l}\text { To check the interface link between the } \\
\text { first page and the login page. }\end{array}$ & $\begin{array}{l}\text { The user clicks the "سجل دخولك" } \\
\text { button. }\end{array}$ & DoItRight directs the user to the login page. & Positive test \\
\hline 3 & $\begin{array}{l}\text { To check the interface link between the } \\
\text { signup page and the start page. }\end{array}$ & $\begin{array}{l}\text { The user enters name and } \\
\text { password, confirms it, and } \\
\text { clicks the "أنشأ حسابك" button. }\end{array}$ & $\begin{array}{l}\text { DoltRight application creates a user account } \\
\text { and directs the user to the start page. }\end{array}$ & Positive test \\
\hline 4 & $\begin{array}{l}\text { To check the interface link between the } \\
\text { login page and the start page }\end{array}$ & $\begin{array}{l}\text { The user enters name and } \\
\text { password and clicks the " دجل button. } \\
\text { سخولك" but }\end{array}$ & $\begin{array}{l}\text { DoItRight application allows the user to } \\
\text { access the app and directs user to the start } \\
\text { page. }\end{array}$ & Positive test \\
\hline 5 & $\begin{array}{l}\text { To check the interface link between the } \\
\text { start page and the first page }\end{array}$ & $\begin{array}{l}\text { The user clicks the " تسجيل "الخروج" button. } \\
\text { تسجيل }\end{array}$ & DoItRight directs the user to the first page. & Positive test \\
\hline 6 & $\begin{array}{l}\text { To check the interface link between the } \\
\text { start page and game page }\end{array}$ & $\begin{array}{l}\text { The user clicks the "ابدأ اللعب" } \\
\text { button. }\end{array}$ & DoItRight directs the user to the game page. & Positive test \\
\hline 7 & $\begin{array}{l}\text { To check the interface link between the } \\
\text { stop page and the start page }\end{array}$ & $\begin{array}{l}\text { The user clicks the "انهاء اللعب" } \\
\text { button. }\end{array}$ & DoItRight directs the user to the start page. & Positive test \\
\hline
\end{tabular}

\section{CONCLUSION AND FUTURE WORK}

To the best of our knowledge, the DoItRight app is the first mobile application aimed at increasing awareness level and intention of Saudi children about littering. The app features meet all user requirements identified and defined during the UCD process such as the potential to educate a child on the proper way to dump litters, motivation, and the application of knowledge gained in real-life. A survey conducted to determine the acceptability of the DoItRight shows that the app has a high acceptability rate among the target audience having recorded a System Usability Scale score of $93.25 \%$.
In the future, we would like to explore the following areas:

i. Conduct a more comprehensive evaluation of the DoItRight app by getting feedback from more children. To get this done, we intend to get approval from the Institutional Review Board (IRB) in Saudi Arabia.

ii. Add more levels to the DoItRight game with varying degrees of difficulty and duration.

iii. Connect the application with the Internet and GPS such that the stages can appear on the map and give a child the feeling of cleaning his current living environment. 
iv. Create a dashboard where users can track their performance with respect to other players using the DoItRight app in their areas.

v. Add multiplayer stages to allow users compete with nearby players and friends.

The DoItRight app can have a significant positive impact on Saudi's public environment if widely adopted among children across the country.

\section{REFERENCES}

[1] Milfont, T. L., \& Schultz, P. W. (2015). Culture and the Natural Environment. Current Opinion in Psychology.

[2] Veiga, J. M., Vlachogianni, T., Pahl, S., Thompson, R. C., Kopke, K., Doyle, T. K., . . . Alampei, I. (2016). Enhancing public awareness and promoting co-responsibility for marine litter in Europe: The challenge of MARLISCO. Marine Pollution Bulletin, 102(2), 309-315. doi:10.1016/j.marpolbul.2016.01.031.

[3] Weaver, R. (2015). Littering in context (s): Using a quasi-natural experiment to explore geographic influences on antisocial behavior. Applied Geography, 57, 142-153.

[4] Yara A. (2017). Extending Understanding of Middle Eastern Littering Behaviour Beyond the Individual: A Formative Research. Griffith Business School. https://doi.org/10.25904/1912/1998.

[5] Yara Almosa, Joy Parkinson \& Sharyn Rundle-Thiele (2020) Preventing Littering: It's Not All about Sticks!, Journal of Nonprofit \& Public Sector Marketing, DOI: 10.1080/10495142.2020.1865236.

[6] Zapata, B., Fernández-Alemán, J., Idri, A. et al. (2015). Empirical Studies on Usability of mHealth Apps: A Systematic Literature Review. J Med Syst 39, 1. https://doi.org/10.1007/s10916-014-0182-2.

[7] Yanghee Kim \& Diantha Smith (2017) Pedagogical and technological augmentation of mobile learning for young children interactive learning environments, Interactive Learning Environments, 25:1, 4-16, DOI: 10.1080/10494820.2015.1087411.

[8] Still, B., \& Crane, K. (2017). Fundamentals of user-centered design: A practical approach. CRC press.

[9] Dopp, A., Parisi, K., Munson, S., and Lyon, A. (2019). A glossary of user-centered design strategies for implementation experts. Translational behavioral medicine, 9(6), 1057-1064.

[10] Mahasneh, R., Romanowski, M. and Basem, R. (2017). Reading social stories in the community: A promising intervention for promoting children's environmental knowledge and behavior in Jordan, The Journal of Environmental Education, 48:5, 334-346, DOI: 10.1080/00958964 .2017.1319789.

[11] Sağlam, M. (2016). Exploring fifth-grade Turkish children's solutions and future plans for environmental pollution through their drawings. Asia-Pacific Forum on Science Learning and Teaching. 17.

[12] Herdiansyah, H, Brotosusilo, A., Negoro, H., Sari, R., and Zakianis, Z. (2021). "Parental Education and Good Child Habits to Encourage
Sustainable Littering Behavior" Sustainability 13, no. 15: 8645. https://doi.org/10.3390/su13158645.

[13] Moqbel, S., El-tah, Z., and Haddad, A. (2020). Anti-littering in developing countries: Motivating the people of Jordan. Waste Management \& Research, 38(7), 726-733. https://doi.org/10.1177/ $0734242 X 19900654$.

[14] Panagiotopoulou, L., Cía Gayarre, N., Scurati, G. W., Etzi, R., Massetti, G., Gallace, A., and Ferrise, F. (2021). "Design of a Serious Game for Children to Raise Awareness on Plastic Pollution and Promoting ProEnvironmental Behaviors." ASME. J. Comput. Inf. Sci. Eng. December 2021; 21(6): 064502. https://doi.org/10.1115/1.4050291.

[15] Rahmayanti, H, Oktaviani, V. and Syani, Y. (2020). Development of sorting waste game android based for early childhood in environmental education. Journal of Physics: Conference Series. 1434. 012029. $10.1088 / 1742-6596 / 1434 / 1 / 012029$.

[16] Kacoroski, J., Liddicoat, K., and Kerlin. S. (2016) Children's use of iPads in outdoor environmental education programs, Applied Environmental Education \& Communication, 15:4, 301-311, DOI: 10.1080/1533015X.2016.1237903.

[17] Safitri, D. and Ika, L., Maksum, A., Nurzengky, I., Marini, A., Zahari, M. and Iskandar, R. (2021). Web-Based Animation Video for Student Environmental Education at Elementary Schools. International Journal of Interactive Mobile Technologies (iJIM). 15. 66. 10.3991/ijim.v1 5 i11.22023.

[18] Buchanan, J., Pressick-Kilborn, K., and Maher, D. (2019). Promoting Environmental Education for Primary School-aged Students Using Digital Technologies. Eurasia Journal of Mathematics, Science and Technology Education, 15(2), em1661. https://doi.org/10.29333/ ejmste/100639.

[19] Kalogiannakis, M. and Papadakis, S. (2017). Combining mobile technologies in environmental education: a Greek case study. International Journal of Mobile Learning and Organisation. 11. 108-130. 10.1504/IJMLO.2017.10005249.

[20] Yu, J., Zhang, J., Chen, Y., Wu, N., Mei, Y., Zhang, D., ... \& Sheng, Y. (2021). A Test Method for Instructional Software of Evaluation and Exercise Based on Mobile Platform. In Journal of Physics: Conference Series. IOP Publishing.

[21] Hendradjaya, B. (2018). A Proposal for New Software Testing Technique for Component Based Software System. International Journal on Electrical Engineering \& Informatics, 10(1).

[22] James R. Lewis (2018) The System Usability Scale: Past, Present, and Future, International Journal of Human-Computer Interaction, 34:7, 577-590, DOI: 10.1080/10447318.2018.1455307.

[23] Kaya, A., Ozturk, R., \& Gumussoy, C. A. (2019). Usability measurement of mobile applications with system usability scale (SUS). In Industrial engineering in the big data era (pp. 389-400). Springer, Cham.

[24] Lewis, J. and Sauro, J. (2019). Item Benchmarks for the System Usability Scale. Journal of Usability Studies. 